\title{
COMMUNICATION
}

\section{A propos d'une névralgie trigéminale atypique révélatrice d'un infarctus cérébelleux occulte.}

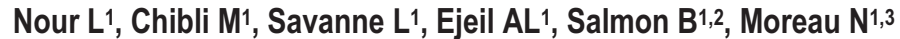

1. Service d'Odontologie, Hôpital Bretonneau - Hôpitaux de Paris (AP-HP)

2. Pathologie, Imagerie et Biothérapies Orofaciales - Université Paris V - Paris Descartes : EA2496

3. Centre de Psychiatrie et Neurosciences - Inserm : U894, Université Paris V - Paris Descartes

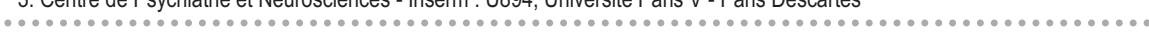

\section{Introduction}

La névralgie trigéminale est une neuropathie oro-faciale extrêmement douloureuse, caractérisée par des crises stéréotypées de "décharges électriques » dans le territoire d'une ou plusieurs branches du nerf trijumeau (Brisman, 2011).

Dans $80 \%$ des cas il n'est retrouvé aucune cause particulière à cette névralgie (hormis un éventuel conflit vasculo-nerveux), dans $20 \%$ des cas, ces névralgies sont secondaires à une compression tumorale au niveau de l'angle ponto-cérébelleux (15\% des cas), une sclérose en plaque voire des causes extrêmement rares comme le syndrome de Wallenberg (Ordas et al., 2011 ; Shulev et al., 2011).

Ainsi, face à une névralgie trigéminale, un bilan étiologique neurologique est indispensable pour écarter une pathologie sous-jacente.

\section{Observation}

II est rapporté le cas d'une patiente de 73 ans, s'étant présentée aux urgences du service d'odontologie de l'hôpital Bretonneau pour des douleurs continues au niveau de la région angulo-mandibulaire droite associées à des crises paroxystiques de douleurs plus intenses lancinantes à type de décharges électriques. Ces crises douloureuses spontanées apparaissaient toutes les 3-4 minutes mais étaient également provoquées par la palpation d'une zone gâchette au niveau de la région angulo-mandibulaire droite, déclenchant des crises stéréotypées accompagnées d'un spasme facial. L'examen neurologique retrouvait une hypoesthésie labiomentonnière droite, sans aucune autre anomalie bucco-dentaire clinique ou radiographique. Par ailleurs, la patiente rapportait des épisodes de tremblements intermittents depuis plusieurs années au niveau des membres du coté droit, non étiquetés (malgré plusieurs explorations neurologiques). Devant ce tableau clinique, il a été émis l'hypothèse d'une névralgie trigéminale droite atypique et prescrit un traitement d'épreuve par Carbamazépine 200 mg/jour. Au rendez-vous de contrôle à une semaine, la patiente rapportait une disparition des décharges électriques et une diminution de l'intensité du fond douloureux continu, confirmant l'hypothèse diagnostique. La patiente a alors été adressée chez un neurologue pour bilan étiologique de cette névralgie trigéminale. L'IRM cérébrale objectiva un hypersignal T2 en séquence FLAIR en bande au niveau de la région cérébelleuse gauche, témoignant d'une séquelle ischémique cérébelleuse ancienne. Une précédente IRM avait été prescrite 3 ans auparavant (pour explorer ses tremblements) et n'avait retrouvée aucune lésion cérébrale ou cérébelleuse à l'examen.

\section{Discussion}

Les névralgies trigéminales peuvent s'accompagner de signes cliniques atypiques (hypoesthésie du V3, fond douloureux inter-critique) qui doivent faire évoquer la possibilité d'une pathologie neurologique sous-jacente. Dans le présent cas, l'IRM cérébrale a permis d'identifier des séquelles d'un infarctus cérébelleux ancien, potentiellement à l'origine des tremblements cérébelleux observés chez cette patiente.

Malgré une chronologie concordante, le tableau clinique de la patiente ne permet pas d'établir formellement un lien de causalité entre l'infarctus cérébelleux et la névralgie trigéminale, bien qu'une lésion ischémique du faisceau trigémino-thalamique puisse être une hypothèse mécanistique envisageable.

\section{Conclusion}

Ce cas de névralgie trigéminale atypique souligne la nécessité d'un bilan étiologique rigoureux, pouvant être à l'origine de la découverte fortuite de pathologies neurologiques occultes.

lisa.nour.lisa@gmail.com

(C) The authors, published by EDP Sciences. This is an Open Access article distributed under the terms of the Creative Commons Attribution License 4.0 (http://creativecommons.org/licenses/by/4.0/). 